UCRHEP-T342

August 2002

\title{
The All-Purpose Neutrino Mass Matrix
}

\author{
Ernest Ma \\ Physics Department, University of California, Riverside, California 92521
}

\begin{abstract}
A four-parameter Majorana neutrino mass matrix is proposed, which is exactly diagonalizable with two mixing angles, one of which is $\pi / 4$ and the other large but less than $\pi / 4$. The form of this mass matrix allows seven realizations of the present data on atmospheric and solar neutrino oscillations: three have the normal hierarchy, two have the inverse hierarchy, and two have three nearly degenerate masses. The possible inclusion of $\mathrm{CP}$ violation is also discussed.
\end{abstract}


With the recent addition of the SNO (Sudbury Neutrino Observatory) neutral-current data [1], the overall picture of solar neutrino oscillations [2] is becoming quite clear. Together with the well-established atmospheric neutrino data [3], the neutrino mixing matrix is now determined to a very good first approximation by

$$
\left(\begin{array}{l}
\nu_{1} \\
\nu_{2} \\
\nu_{3}
\end{array}\right)=\left(\begin{array}{ccc}
\cos \theta & \sin \theta / \sqrt{2} & \sin \theta / \sqrt{2} \\
-\sin \theta & \cos \theta / \sqrt{2} & \cos \theta / \sqrt{2} \\
0 & -1 / \sqrt{2} & 1 / \sqrt{2}
\end{array}\right)\left(\begin{array}{c}
\nu_{e} \\
\nu_{\mu} \\
\nu_{\tau}
\end{array}\right),
$$

where $\nu_{1,2,3}$ are Majorana neutrino mass eigenstates. In the above, $\sin ^{2} 2 \theta_{\text {atm }}=1$ is already assumed and $\theta$ is the solar mixing angle which is now known to be large but not maximal [4], i.e. $\tan ^{2} \theta \simeq 0.4$. The $U_{e 3}$ entry has been assumed zero but it is only required to be small [5], i.e. $\left|U_{e 3}\right|<0.16$. A nonzero $U_{e 3}$ with a possible complex phase for CP violation will be discussed later.

Denoting the masses of $\nu_{1,2,3}$ as $m_{1,2,3}$, the solar neutrino data [1, 2] require that $m_{2}^{2}>m_{1}^{2}$ with $\theta<\pi / 4$, and in the case of the favored large-mixing-angle solution [4],

$$
\Delta m_{\text {sol }}^{2}=m_{2}^{2}-m_{1}^{2} \simeq 5 \times 10^{-5} \mathrm{eV}^{2}
$$

The atmospheric neutrino data [3] require

$$
\left|m_{3}^{2}-m_{1,2}^{2}\right| \simeq 2.5 \times 10^{-3} \mathrm{eV}^{2}
$$

without deciding whether $m_{3}^{2}>m_{1,2}^{2}$ or $m_{3}^{2}<m_{1,2}^{2}$.

The big question now is what the neutrino mass matrix itself should look like. It is proposed here that it should be of the form

$$
\mathcal{M}_{\nu}=\left(\begin{array}{ccc}
a+2 b+2 c & d & d \\
d & b & a+b \\
d & a+b & b
\end{array}\right)
$$

This matrix has 4 parameters which are assumed first to be all real. In that case, it is exactly diagonalized by Eq. (1) with its eigenvalues exactly given by (I):

$$
m_{1}=a+2 b+c-\sqrt{c^{2}+2 d^{2}}
$$




$$
\begin{aligned}
& m_{2}=a+2 b+c+\sqrt{c^{2}+2 d^{2}} \\
& m_{3}=-a,
\end{aligned}
$$

with

$$
\tan \theta=\frac{\sqrt{2} d}{\sqrt{c^{2}+2 d^{2}}-c},
$$

for $a+2 b+c>0, c<0$, and (II):

$$
\begin{aligned}
& m_{1}=a+2 b+c+\sqrt{c^{2}+2 d^{2}}, \\
& m_{2}=a+2 b+c-\sqrt{c^{2}+2 d^{2}}, \\
& m_{3}=-a,
\end{aligned}
$$

with

$$
\tan \theta=\frac{\sqrt{c^{2}+2 d^{2}}-c}{\sqrt{2} d}
$$

for $a+2 b+c<0, c>0$.

Note that $\theta$ depends only on the ratio $d / c$, which must be of order unity. This shows the advantage for adopting the parametrization of Eq. (4). The constraints of Eqs. (2) and (3) are then realized by the following 7 different conditions on $a, b$, and $c$.

(1) ||$a+2 b+c\left|-\sqrt{c^{2}+2 d^{2}}\right|<<|a+2 b+c|<<|a|$, i.e. $\left|m_{1}\right|<<\left|m_{2}\right|<<\left|m_{3}\right|$.

(2) $\sqrt{c^{2}+2 d^{2}}<<|a+2 b+c|<<|a|$, i.e. $\left|m_{1}\right| \simeq\left|m_{2}\right|<<\left|m_{3}\right|$.

(3) $|a+2 b+c|<<\sqrt{c^{2}+2 d^{2}}<<|a|$, i.e. $\left|m_{1}\right| \simeq\left|m_{2}\right|<<\left|m_{3}\right|$.

(4) $|a|, \sqrt{c^{2}+2 d^{2}}<<|a+2 b+c|$, i.e. $\left|m_{3}\right|<<\left|m_{1}\right| \simeq\left|m_{2}\right|$.

(5) $|a|,|a+2 b+c|<<\sqrt{c^{2}+2 d^{2}}$, i.e. $\left|m_{3}\right|<<\left|m_{1}\right| \simeq\left|m_{2}\right|$.

(6) $\sqrt{c^{2}+2 d^{2}}<<|| a+2 b+c|-| a||<<|a|$, i.e. $\left|m_{1}\right| \simeq\left|m_{2}\right| \simeq\left|m_{3}\right|$.

(7) $|a+2 b+c|<<\sqrt{c^{2}+2 d^{2}} \simeq|a|$, i.e. $\left|m_{1}\right| \simeq\left|m_{2}\right| \simeq\left|m_{3}\right|$. 
Cases (1) to (3) have the normal hierarchy. Cases (4) and (5) have the inverse hierarchy. Cases (6) and (7) have 3 nearly degenerate masses. The versatility of Eq. (4) has clearly been demonstrated.

The above 7 cases encompass all models of the neutrino mass matrix that have ever been proposed which also satisfy Eq. (1). They are also very useful for discussing the possibility of neutrinoless double beta $\left(\beta \beta_{0 \nu}\right)$ decay in the context of neutrino oscillations [6]. The effective mass $m_{0}$ measured in $\beta \beta_{0 \nu}$ decay is $|a+2 b+2 c|$. However, neutrino oscillations constrain $|a+2 b+c|$ and $\sqrt{c^{2}+2 d^{2}}$, as well as $|d / c|$. Using

$$
|a+2 b+2 c|=|| a+2 b+c| \pm| c||=|| a+2 b+c\left| \pm \cos 2 \theta \sqrt{c^{2}+2 d^{2}}\right|
$$

the following conditions on $m_{0}$ are easily obtained:

$$
\begin{aligned}
& \text { (1) } m_{0} \simeq \sin ^{2} \theta\left|m_{2}\right| \simeq \sin ^{2} \theta \sqrt{\Delta m_{\text {sol }}^{2}} \\
& \text { (2) } \quad m_{0} \simeq\left|m_{1,2}\right|<<\sqrt{\Delta m_{\text {atm }}^{2}} \\
& \text { (3) } m_{0} \simeq \cos 2 \theta\left|m_{1,2}\right|<<\cos 2 \theta \sqrt{\Delta m_{\text {atm }}^{2}}, \\
& \text { (4) } m_{0} \simeq \sqrt{\Delta m_{\text {atm }}^{2}} \\
& \text { (5) } m_{0} \simeq \cos 2 \theta \sqrt{\Delta m_{\text {atm }}^{2}} \\
& \text { (6) } m_{0} \simeq\left|m_{1,2,3}\right| \\
& \text { (7) } m_{0} \simeq \cos 2 \theta\left|m_{1,2,3}\right| .
\end{aligned}
$$

If $m_{0}$ is measured [7] to be significantly larger than $0.05 \mathrm{eV}$, then only Cases (6) and (7) are allowed. However, as Eqs. (19) and (20) show, the true mass of the three neutrinos is still subject to a two-fold ambiguity, which is a well-known result.

The underlying symmetry of Eq. (4) which results in $U_{e 3}=0$ is its invariance under the interchange of $\nu_{\mu}$ and $\nu_{\tau}$. Its mass eigenstates are then separated according to whether they are even $\left(\nu_{1,2}\right)$ or odd $\left(\nu_{3}\right)$ under this interchange, as shown by Eq. (1). To obtain $U_{e 3} \neq 0$, 
this symmetry has to be broken. One interesting possibility is to rewrite Eq. (4) as

$$
\mathcal{M}_{\nu}=\left(\begin{array}{ccc}
a+2 b+2 c & d & d^{*} \\
d & b & a+b \\
d^{*} & a+b & b
\end{array}\right)
$$

where $a, b, c$ are real but $d$ is complex. This reduces to Eq. (4) if $I m d=0$, but if $\operatorname{Imd} \neq 0$, then $U_{e 3} \neq 0$.

To obtain $U_{e 3}$ in a general way, first rotate to the basis spanned by $\nu_{e},\left(\nu_{\mu}+\nu_{\tau}\right) / \sqrt{2}$, and $\left(\nu_{\tau}-\nu_{\mu}\right) / \sqrt{2}$, i.e.

$$
\mathcal{M}_{\nu}=\left(\begin{array}{ccc}
a+2 b+2 c & \sqrt{2} \text { Red } & -\sqrt{2} i \text { Imd } \\
\sqrt{2} \text { Red } & a+2 b & 0 \\
-\sqrt{2} i \text { Imd } & 0 & -a
\end{array}\right)
$$

Whereas $\mathcal{M}_{\nu}$ is diagonalized by

$$
U \mathcal{M}_{\nu} U^{T}=\left(\begin{array}{ccc}
m_{1} & 0 & 0 \\
0 & m_{2} & 0 \\
0 & 0 & m_{3}
\end{array}\right)
$$

$\mathcal{M}_{\nu} \mathcal{M}_{\nu}^{\dagger}$ is diagonalized by

$$
U\left(\mathcal{M}_{\nu} \mathcal{M}_{\nu}^{\dagger}\right) U^{\dagger}=\left(\begin{array}{ccc}
\left|m_{1}\right|^{2} & 0 & 0 \\
0 & \left|m_{2}\right|^{2} & 0 \\
0 & 0 & \left|m_{3}\right|^{2}
\end{array}\right)
$$

Here

$$
\mathcal{M}_{\nu} \mathcal{M}_{\nu}^{\dagger}=\left(\begin{array}{ccc}
(a+2 b+2 c)^{2}+2|d|^{2} & 2 \sqrt{2}(a+2 b+c) \text { Red } & 2 \sqrt{2} i(a+b+c) \text { Imd } \\
2 \sqrt{2}(a+2 b+c) \text { Red } & (a+2 b)^{2}+2(\text { Red })^{2} & 2 i \text { RedImd } \\
-2 \sqrt{2} i(a+b+c) \text { Imd } & -2 i \text { RedImd } & a^{2}+2(\text { Imd })^{2}
\end{array}\right) .
$$

To obtain $U_{e 3}$ for small $I m d$, consider the matrix

$$
A=\mathcal{M}_{\nu} \mathcal{M}_{\nu}^{\dagger}-\left[a^{2}+2(\operatorname{Imd})^{2}\right] I
$$

where $I$ is the identity matrix. Now $A$ is diagonalized by $U$ as well and $U_{e 3}$ is simply given by

$$
U_{e 3} \simeq \frac{A_{e 3}}{A_{e e}}=\frac{2 \sqrt{2} i(a+b+c) I m d}{(a+2 b+2 c)^{2}-a^{2}+2(\text { Red })^{2}}
$$


to a very good approximation and leads to

$$
\begin{aligned}
(1),(2),(3) \quad U_{e 3} & \simeq \frac{-\sqrt{2} i \operatorname{Imd}}{a} \\
(4),(6) \quad U_{e 3} & \simeq \frac{i \operatorname{Imd}}{\sqrt{2} b} \\
(5) & U_{e 3} \simeq \frac{\sqrt{2} i \operatorname{Imd}}{c} \\
(7) & U_{e 3} \simeq \frac{\sqrt{2} i(a+c) \operatorname{Imd}}{c^{2}-a^{2}+2(\text { Red })^{2}}
\end{aligned}
$$

In all cases, the magnitude of $U_{e 3}$ can be as large as the present experimental limit [5] of 0.16 and its phase is $\pm \pi / 2$. Thus the $\mathrm{CP}$ violating effect in neutrino oscillations is predicted to be maximal by Eq. (21), which is a very desirable scenario for future long-baseline neutrino experiments.

The above analysis shows that for $U_{e 3}=0$ and $\sin ^{2} 2 \theta_{a t m}=1$, the seven cases considered cover all possible patterns of the $3 \times 3$ Majorana neutrino mass matrix, as indicated by present atmospheric and solar neutrino data. Any successful model should predict Eq. (4) at least as a first approximation. One such example already exists [8], where $b=c=d=0$ corresponds to the non-Abelian discrete symmetry $A_{4}$, i.e. the finite group of the rotations of a regular tetrahedron. This leads to Case (6), i.e. three nearly degenerate masses, with the common mass equal to that measured in $\beta \beta_{0 \nu}$ decay. It has also been shown recently 9] that starting with this pattern, the correct mass matrix, i.e. Eq. (21) with the complex phase in the right place, is automatically obtained with the most general application of radiative corrections. In particular, if soft supersymmetry breaking is assumed to be the origin of these radiative corrections, then the neutrino mass matrix is correlated with flavor violation in the slepton sector, and may be tested in future collider experiments.

If $m_{0}$ turns out to be of order $\sqrt{\Delta m_{a t m}^{2}}$ or less, then Cases (6) and (7) would be ruled out, and further progress in understanding the pattern of neutrino oscillations will depend on having a rationale for choosing one of the remaining 5 cases. Consider for example Case 
(1) with $a+2 b+c=\sqrt{c^{2}+2 d^{2}}$ and $c=-d / 2<0$, then Eq. (4) becomes

$$
\mathcal{M}_{\nu}=\left(\begin{array}{ccc}
d & d & d \\
d & b & -b+2 d \\
d & -b+2 d & b
\end{array}\right),
$$

which results in $m_{1}=0, m_{2}=3 d, m_{3}=2 b-2 d$, and $\tan ^{2} \theta=0.5$.

As another example, consider Case (4) with $a=0, c=-d / 2<0$, then Eq. (4) becomes

$$
\mathcal{M}_{\nu}=\left(\begin{array}{ccc}
2 b-d & d & d \\
d & b & b \\
d & b & b
\end{array}\right)
$$

which results in $m_{1}=2 b-2 d, m_{2}=2 b+d, m_{3}=0$, and $\tan ^{2} \theta=0.5$. Thus $m_{2}^{2}-m_{1}^{2} \simeq 12 d b$ and $m_{1,2}^{2}-m_{3}^{2} \simeq 4 b^{2}$, where $d<<b$ has been used.

Both examples are simple acceptable two-parameter solutions of the present atmospheric and solar neutrino data. This shows the utility of Eq. (4) as a way to find possible patterns which may even turn out to be supported by some symmetry. As further examples, the case $c=0$ was discussed in Ref. [10] and the case $a+2 b+c=0$ was discussed in Ref. [11].

In conclusion, recent experimental progress on neutrino oscillations points to a neutrino mixing matrix which can be understood in a systematic way in terms of an all-purpose neutrino mass matrix, i.e. Eq. (4), and its simple extension, i.e. Eq. (21), to allow for a nonzero and imaginary $U_{e 3}$, i.e. Eq. (27). Seven possible cases have been identified, each with a different prediction for $\beta \beta_{0 \nu}$ decay, i.e. Eqs. (14) to (20). The present analysis should be useful as both a checklist and a roadmap for the further theoretical understanding of the origin of neutrino masses and mixing.

This work was supported in part by the U. S. Department of Energy under Grant No. DEFG03-94ER40837. 


\section{References}

[1] Q. R. Ahmad et al., SNO Collaboration, Phys. Rev. Lett. 89, 011301, 011302 (2002).

[2] S. Fukuda et al., Super-Kamiokande Collaboration, Phys. Rev. Lett. 86, 5656 (2001) and references therein; Q. R. Ahmad et al., SNO Collaboration, Phys. Rev. Lett. 87, 071301 (2001).

[3] S. Fukuda et al., Super-Kamiokande Collaboration, Phys. Rev. Lett. 85, 3999 (2000) and references therein.

[4] V. Barger et al., Phys. Lett. B537, 179 (2002); A. Bandyopadhyay et al., Phys. Lett. B540, 14 (2002); J. N. Bahcall et al., hep-ph/0204314; P. Aliani et al., hepph/0205053; P. C. de Holanda and A. Yu. Smirnov, hep-ph/0205241; A. Strumia et al., hep-ph/0205261; G. L. Fogli et al., hep-ph/0206162.

[5] M. Apollonio et al., Phys. Lett. B466, 415 (1999); F. Boehm et al., Phys. Rev. D64, 112001 (2001).

[6] See for example H. V. Klapdor-Kleingrothaus and U. Sarkar, Phys. Lett. B532, 71 (2002); S. Pascoli and S. T. Petcov, hep-ph/0205022; and references therein.

[7] Evidence for $m_{0}$ in the range 0.11 to $0.56 \mathrm{eV}$ has been claimed by H. V. KlapdorKleingrothaus et al., Mod. Phys. Lett. A16, 2409 (2001). See also comments by C. E. Aalseth et al., hep-ex/0202018, and the reply by H. V. Klapdor-Kleingrothaus, hep$\mathrm{ph} / 0205228$.

[8] E. Ma and G. Rajasekaran, Phys. Rev. D64, 113012 (2001); E. Ma, Mod. Phys. Lett. A17, 289 (2002); E. Ma, Mod. Phys. Lett. A17, 627 (2002).

[9] K. S. Babu, E. Ma, and J. W. F. Valle, hep-ph/0206292. 
[10] R. N. Mohapatra and S. Nussinov, Phys. Rev. D60, 013002 (1999).

[11] A. Joshipura, hep-ph/0205038 (Phys. Lett. B, in press). 